This paper has been published in Science China Mathematics, 55(5):961-966 (2012).

Copyright 2012 by Science China Press and Springer-Verlag.

The final publication is available at www.springerlink.com.

http://link.springer.com/article/10.1007/s11425-011-4356-9

http://dx.doi.org/10.1007/s11425-011-4356-9 


\title{
A question on partial CAP-subgroups of finite groups
}

\author{
A. Ballester-Bolinches* R. Esteban-Romero ${ }^{\dagger}$ \\ Yangming $\mathrm{Li}^{\ddagger}$
}

\begin{abstract}
A subgroup $H$ of a finite group $G$ is a partial CAP-subgroup of $G$ if there is a chief series of $G$ such that $H$ either covers or avoids every chief factor of the series. The structural impact of the partial cover and avoidance property of some distinguished subgroups of a group has been studied by many authors. However there are still some open questions which deserve an answer. The purpose of the present paper is to give a complete answer to one of these questions.

Keywords: finite groups, minimal subgroups, partial cover and avoidance property, saturated formations.

Mathematics Subject Classification (2000): 20D10, 20 D20.
\end{abstract}

\section{Introduction}

In this paper all groups are assumed to be finite. A subgroup $A$ of a group $G$ is said to have the cover-avoidance property in $G$ and is called a $C A P$-subgroup of $G$ if either $H A=K A$ or $A \cap H=A \cap K$ for every chief factor $H / K$ of $G$. CAP-subgroups have played an important role in the structural study of soluble groups because, in that universe, some important subgroups such as Hall subgroups, maximal subgroups, normalisers associated to saturated formations and prefrattini subgroups enjoy the property. In fact, as it is shown in [1, Chapter 4], the cover and avoidance property and conjugacy of

*Departament d'Àlgebra, Universitat de València, Dr. Moliner, 50, E-46100 Burjassot, València, Spain, email: Adolfo.Ballester@uv.es

†Institut Universitari de Matemàtica Pura i Aplicada, Universitat Politècnica de València, Camí de Vera, s/n, E-46022 València, Spain, email: resteban@mat.upv.es

${ }^{\ddagger}$ Department of Mathematics, Guangdong University of Education, Guangzhou, 510310, People's Republic of China; email: liyangming@gdei.edu.cn 
the members of some of the above families characterise solubility. Despite of this, there was a little evidence to suggest a huge proliferation of results in the area. However, Ezquerro [5] began to study the structural impact of the cover and avoidance embedding of some relevant families of subgroups. He observed that it is enough to impose the cover and avoidance property to the maximal subgroups of the Sylow subgroups to get supersolubility. This result establishes the standpoint for a research project consisting in characterising some formations by the cover and avoidance property of some distinguished subgroups such as maximal subgroups of Sylow subgroups, second maximal subgroups of Sylow subgroups or minimal subgroups. For an overview, the reader is referred to $[2,7,11]$ and the papers cited therein.

Our point of departure is the observation that the cover and avoidance property is not persistent in intermediate subgroups, that is, if $H$ is a CAPsubgroup of $G$ and $H$ is contained in $K$, then $H$ is not a CAP-subgroup of $K$ in general (cf. [2, Example 1.3]). Surprisingly, the following weaker property introduced by Y. Fan, X. Guo, and K. P. Shum in [6] has this property: a subgroup $A$ of a group $G$ is said to be partial $C A P$-subgroup of $G$ if there exists a chief series $\Gamma_{A}$ of $G$ such that $A$ either covers or avoids each factor of $\Gamma_{A}$. These type of subgroups are also called semi CAP-subgroups (see [6]) or SCAP-subgroups (see [11]). Of course, every subgroup of a supersoluble group is a partial CAP-subgroup. Hence the natural question arising is whether there are more restricted families of subgroups whose partial cover and avoidance property could guarantee supersolubility. In [2] and [11] it is proved, as a particular case of a more general result, that the partial CAPproperty of a much more restricted family of subgroups, namely the maximal subgroups of the Sylow subgroups of the generalised Fitting subgroup, implies supersolubility. In addition, in [2] a local approach is used. It allows us to discover new situations and see how the global properties can be deduced from the local ones.

A further step in this analysis is to consider a dual family, that is, the one of all subgroups of order $p$ or order 4 , and wonder whether a group is supersoluble provided that all the members of this family are partial CAPsubgroups. In fact, the following more general question was asked by the third author in [10]:

Question 1.1. Let $\mathfrak{F}$ be a saturated formation containing $\mathfrak{U}$, the class of all supersoluble groups, and $H$ a normal subgroup of a group $G$ such that $G / H \in \mathfrak{F}$. Suppose that, for every non-cyclic Sylow subgroup $P$ of $\mathrm{F}^{*}(H)$, $P$ has a subgroup $D$ such that $1<|D|<|P|$ and all subgroups $E$ of $P$ with order $|E|=|D|$ and with order $|E|=2|D|$ (if $P$ is a non-abelian 2-group and $|P: D|>2$ ) are partial CAP-subgroups of $G$. Does $G$ belong to $\mathfrak{F}$ ? 
The following example gives a negative answer to Question 1.1.

Example 1.2. Let $H=\left\langle a, b \mid a^{5}=b^{5}=1, a b=b a\right\rangle$ be an elementary abelian group of order $5^{2}$, and let $\alpha$ be an automorphism of $H$ of order 3 satisfying that $a^{\alpha}=b, b^{\alpha}=a^{-1} b^{-1}$. Let $H_{1}, H_{2}=\left\langle a^{\prime}, b^{\prime}\right\rangle$ be two copies of $H$ and denote by $G=\left[H_{1} \times H_{2}\right]\langle\alpha\rangle$ the corresponding semidirect product. If $A$ is a subgroup of $G$ of order 25, there exists a minimal normal subgroup $N$ such that $A \cap N=1$. Hence $A$ covers or avoids the following chief series of $G$ :

$$
1<N<A N<G
$$

Consequently $A$ is a partial CAP-subgroup of $G$ and so $G$ is a non-supersoluble group whose every second maximal subgroups are partial CAPsubgroups. Note that $H$ is an absolutely irreducible $C_{3}$-module over the finite field of 5-elements.

In fact, the main result of [3] characterises when the partial cover and avoidance property of the second maximal subgroups implies supersolubility.

The main objective of the present paper is to show that Question 1.1 has an affirmative answer for minimal subgroups.

Theorem 1.3. Let $\mathfrak{F}$ be a saturated formation containing $\mathfrak{U}$, the class of all supersoluble groups, and let $G$ be a group with a normal subgroup $H$ such that $G / H \in \mathfrak{F}$. Then $G \in \mathfrak{F}$ if every cyclic subgroup of $\mathrm{F}^{*}(H)$ of prime order or order 4 is a partial CAP-subgroup of $G$.

We note that the saturation of $\mathfrak{F}$ cannot be removed in Theorem 1.3. Consider the formation $\mathfrak{F}$ of all groups whose supersoluble residual is trivial or a direct product of copies of the alternating group $S=A_{5}$ of degree 5 . Then $\mathfrak{F}$ is a formation which contains the class $\mathfrak{U}$ of all supersoluble groups. The group $G=\mathrm{SL}_{2}(5)$ has a normal subgroup $H=\mathrm{Z}(G)$ of order 2 and obviously $H$, which is the unique subgroup of prime order of $\mathrm{F}^{*}(H)$, is a partial CAP-subgroup of $G$. However, $G$ does not belong to $\mathfrak{F}$.

The proof of the above result depends on the following local theorem.

Theorem 1.4. Let $p$ be a prime and let $G$ be a group. If every cyclic subgroup of $G$ of order $p$ or order 4 is a partial CAP-subgroup of $G$, then $G$ is $p$ supersoluble.

As a consequence of Theorem 1.3, we get another local result.

Corollary 1.5. Assume that $p$ is a prime and $\mathfrak{F}$ is a saturated formation containing all p-supersoluble groups such that $\mathfrak{E}_{p^{\prime}} \mathfrak{F}=\mathfrak{F}$. Suppose that $G$ is a group with a normal subgroup $N$ such that $G / N$ belongs to $\mathfrak{F}$. If every cyclic subgroup of $\mathrm{F}_{p}^{*}(N)$ of order $p$ or 4 is a partial $C A P$-subgroup of $G$, then $G$ belongs to $\mathfrak{F}$. 
Recall that for every group $X$ and for every prime $p$, the $\operatorname{subgroup} \mathrm{F}_{p}^{*}(X)$ is defined to be the subgroup of $X$ such that $\mathrm{F}_{p}^{*}(X) / \mathrm{O}_{p^{\prime}}(X)=\mathrm{F}^{*}\left(X / \mathrm{O}_{p^{\prime}}(X)\right)$.

\section{Preliminaries}

This section contains the results which are needed to prove our main theorems. We begin with a lemma containing the basic properties of the partial CAP-subgroups which turn out very useful in induction arguments.

Lemma 2.1 (see [6]). Every CAP-subgroup of $G$ is a partial CAP-subgroup of $G$. Furthermore, if $S$ is a partial CAP-subgroup of a group $G$, then:

1. If $S \leq K \leq G$, then $S$ is a partial CAP-subgroup of $K$.

2. If $N \leq S$ and $N \unlhd G$, then $S / N$ is a partial CAP-subgroup of $G / N$.

3. If $N \unlhd G$ and $(|S|,|N|)=1$, then $S N / N$ is a partial CAP-subgroup of $G / N$.

The next lemma describes a configuration often encountered in the study of partial CAP-subgroups. Although its proof is part of the proof of Lemma 2.2 in [2], we include it here for the sake of completeness.

Lemma 2.2. Let $H$ be a partial CAP-subgroup of a group $G$. Suppose that $Q$ is a normal subgroup of $G$ such that $H$ is contained in $Q$. Then there exists a chief series $\Omega_{H}$ of $G$ passing through $Q$ such that $H$ either covers or avoids each chief factor in $\Omega_{H}$.

Proof. Since $H$ is a partial CAP-subgroup of the group $G$, there exists a chief series

$$
\Gamma_{H}: 1=G_{0}<G_{1}<\cdots<G_{n}=G
$$

of $G$ such that $H$ either covers or avoids each chief factor in $\Gamma_{H}$. Since $Q$ is a normal subgroup of $G$, then $Q$ is a CAP-subgroup of $G$. Therefore

$$
\Gamma_{H} \cap Q: 1=G_{0} \cap Q<G_{1} \cap Q<\cdots<G_{n} \cap Q=Q
$$

is, avoiding repetitions, part of a chief series of $G$. Moreover, if $H$ covers (respectively, avoids) $G_{i+1} / G_{i}$, then $H$ covers (respectively, avoids) $\left(G_{i+1} \cap\right.$ $Q) /\left(G_{i} \cap Q\right)$.

We can complete $\Gamma_{H} \cap Q$ to obtain a chief series $\Omega_{H}$ of $G$. Note that $H$ avoids all chief factors above $Q$. Hence there exits a chief series

$$
\Omega_{H}: 1=G_{0}^{*}<G_{1}^{*}<\cdots<G_{r}^{*}=Q<G_{r+1}^{*}<\cdots<G_{n}^{*}=G
$$

of $G$ such that $H$ covers or avoids all chief factors of $G$ in $\Omega_{H}$. 
Recall that a class of groups $\mathfrak{F}$ is called a formation if it is closed under taking epimorphic images and subdirect products. $\mathfrak{F}$ is said to be saturated if $G / \Phi(G) \in \mathfrak{F}$ implies $G \in \mathfrak{F}$.

Let $\mathfrak{F}$ be a non-empty formation. Each group $G$ has a smallest normal subgroup whose quotient belongs to $\mathfrak{F}$; this is called the $\mathfrak{F}$-residual of $G$ and it is denoted by $G^{\mathfrak{F}}$. Clearly $G^{\mathfrak{F}}$ is a characteristic subgroup of $G$ (cf. [1, 4] for details).

Let $p$ be a prime. A group $G$ is said to be $p$-supersoluble if $G$ is $p$-soluble and every chief factor of order divisible by $p$ is cyclic. The class $\mathfrak{U}_{p}$ of all $p$-supersoluble groups is a saturated formation. Clearly the intersection of all $\mathfrak{U}_{p}$ is again a saturated formation which is composed of all soluble groups whose chief factors are cyclic. This class is the class of all supersoluble groups and is denoted by $\mathfrak{U}$.

Let $\mathfrak{H}$ be a non-empty class of groups. According to $[1,1.2 .9,2.3 .18]$, a chief factor $H / K$ of a group $G$ is said to be $\mathfrak{H}$-central in $G$ if $[H / K] * G$ belongs to $\mathfrak{H}$, where $[H / K] * G$ is the semidirect product $[H / K]\left(G / \mathrm{C}_{G}(H / K)\right)$ if $H / K$ is abelian and $G / \mathrm{C}_{G}(H / K)$ if $H / K$ is non-abelian. A normal subgroup $N$ of a group $G$ is called $\mathfrak{H}$-hypercentral in $G$ if every chief factor of $G$ below $N$ is $\mathfrak{H}$-central in $G$. By virtue of the generalised Jordan-Hölder theorem $[1,1.2 .36]$, we obtain that the product of $\mathfrak{H}$-hypercentral normal subgroups of a group $G$ is again $\mathfrak{H}$-hypercentral in $G$. Thus every group $G$ possesses a unique maximal normal $\mathfrak{H}$-hypercentral subgroup called the $\mathfrak{H}$-hypercentre of $G$ and denoted by $\mathrm{Z}_{\mathfrak{H}}(G)$. Applying again the generalised Jordan-Hölder theorem, every chief factor of $G$ below $\mathrm{Z}_{\mathfrak{H}}(G)$ is $\mathfrak{H}$-central in $G$.

The following theorem is a good illustration of how the partial cover and avoidance property of the minimal subgroups influences the embedding of a normal $p$-subgroup and it plays a crucial part in the proof of our main results.

Theorem 2.3. Let $p$ be a prime and let $P$ be a normal $p$-subgroup of a group $G$. If every cyclic subgroup of $P$ of order $p$ or 4 is a partial CAP-subgroup of $G$, then $P$ is contained in $\mathrm{Z}_{\mathfrak{U}}(G)$.

Proof. Suppose, by way of contradiction, that the theorem is false, and choose a pair $(G, P)$ for which it fails. Then there exists a chief factor of $G$ below $P$ which is not of prime order. Among the non-cyclic chief factors of $G$ below $P$, we choose one $L / K$ with $|L|$ as small as possible.

Assume there exists an element $x$ of $L$ of prime order or order 4 which is not in $K$. Then $\langle x\rangle$ is a partial CAP-subgroup of $G$. Applying Lemma 2.2, there exists a chief series

$$
\Gamma: 1=L_{0}<L_{1}<\cdots<L_{s-1}<L_{s}=L<\cdots<G
$$


of $G$ passing through $L$ such that $\langle x\rangle$ either covers or avoids each chief factor in $\Gamma$. By the choice of $L / K$, we have each chief factor $L_{i} / L_{i-1}$ is of prime order for $i=1,2, \ldots, s-1$. If $\langle x\rangle$ covers $L_{s} / L_{s-1}$, then $L_{s} / L_{s-1}$ is of prime order. Hence $L \leq \mathrm{Z}_{\mathfrak{U}}(G)$. This implies that $L / K$ is of prime order, which is not the case. Therefore $\langle x\rangle$ avoids $L_{s} / L_{s-1}$. Then $x \in L_{s-1}$ and so $L_{s-1} K>K$. Hence $L=L_{s-1} K$. Since $L / K=L_{s-1} K / K$ is $G$-isomorphic to $L_{s-1} /\left(L_{s-1} \cap\right.$ $K)$, it follows that $L_{s-1} /\left(L_{s-1} \cap K\right)$ is a chief factor of $G$. The choice of $L / K$ implies that $L_{s-1} /\left(L_{s-1} \cap K\right)$ is of prime order. Consequently $L / K$ is of prime order. This contradiction proves that every element of $L$ of order $p$ or order 4 is contained in $K$.

Let $X$ denote the intersection of the centralisers of the chief factors of $G$ below $K$. Then $X$ stabilises a chain of subgroups of $K$. Applying [4, A, 12.4], $\mathrm{O}^{p}(X)$ centralises $K$. In particular, $\mathrm{O}^{p}(X)$ centralises every element of prime order or order 4 of $L$. By [8, IV, 5.12], $\mathrm{O}^{p}(X)$ centralises $L$. Thus $X / \mathrm{C}_{X}(L / K)$ is a normal $p$-subgroup of $G / \mathrm{C}_{G}(L / K)$. By [4, B, 3.12], $X$ centralises $L / K$. This implies that $L / K$ can be regarded as an irreducible $G / X$-module over the finite field of $p$-elements. Note that every chief factor $U / V$ of $G$ below $K$ is of order $p$ and so $G / \mathrm{C}_{G}(U / V)$ is cyclic of order dividing $p-1$. Consequently, $G / X$ is abelian of exponent dividing $p-1$. Applying $[4, \mathrm{~B}, 9.8], L / K$ has order $p$. This final contradiction shows that no such counterexample $G$ exists.

Since every cyclic chief factor of order 2 in a given chief series of a group is central, we have:

Corollary 2.4. Suppose that $P$ is a normal 2-subgroup of $G$. If every cyclic subgroup of $P$ of order 2 or 4 is a partial CAP-subgroup of $G$, then $P \leq$ $\mathrm{Z}_{\infty}(G)$, the nilpotent hypercentre of $G$.

\section{Proofs of the main theorems}

We are now ready to prove our main results.

Proof of Theorem 1.4. Suppose that the theorem is false, and let $G$ be a counterexample of minimal order. The structure of $G$ is analysed, and eventually a contradiction is reached. For the ease of reading we break the argument into separately-stated steps.

Step 1. Every proper subgroup of $G$ is p-supersoluble, that is, $G$ is a minimal non-p-supersoluble group.

Let $M$ be a maximal subgroup of $G$ and let $L$ be a cyclic subgroup of $M$ of order $p$ or order 4 . Then $L$ is a partial CAP-subgroup of $G$. By 
Lemma 2.1 (1), $L$ is a partial CAP-subgroup of $M$. Hence $M$ satisfies the hypotheses of the theorem. The minimal choice of $G$ yields that $M$ is $p$ supersoluble. Consequently, every proper subgroup of $G$ is $p$-supersoluble, since the class of all $p$-supersoluble groups is subgroup-closed. This is to say that $G$ is a minimal non- $p$-supersoluble group.

Step 2. $\mathrm{O}_{p^{\prime}}(G)=1$. Therefore $\mathrm{F}(G)=\mathrm{O}_{p}(G)$.

Set $\bar{G}=G / \mathrm{O}_{p^{\prime}}(G)$. Suppose that $\bar{L}$ is a cyclic subgroup of $\bar{G}$ of order $p$ or 4 . Then we can write $\bar{L}=L \mathrm{O}_{p^{\prime}}(G) / \mathrm{O}_{p^{\prime}}(G)$, where $L$ is a cyclic subgroup of $G$ of order $p$ or 4 . By hypothesis, $L$ is a partial CAP-subgroup of $G$. By Lemma 2.1 (3), $\bar{L}$ is a partial CAP-subgroup of $\bar{G}$. Hence $\bar{G}$ satisfies the hypothesis of the theorem. If $\mathrm{O}_{p^{\prime}}(G)$ were non-trivial, then $\bar{G}$ would be a $p$-supersoluble group by the minimal choice of $G$. In this case, $G$ would be a $p$-supersoluble group. This contradicts Step 1. Hence $\mathrm{O}_{p^{\prime}}(G)=1$.

Applying Theorem 2.3, we have:

Step 3. $\mathrm{F}(G) \leq \mathrm{Z}_{\mathfrak{U}}(G)$.

Step 4. $G=\mathrm{F}^{*}(G)$.

Assume that $\mathrm{F}^{*}(G)$ is a proper subgroup of $G$. Then $\mathrm{F}^{*}(G)$ is $p$-supersoluble and so $\mathrm{F}^{*}(G)=\mathrm{F}(G)=\mathrm{O}_{p}(G)$. Since $\mathrm{F}(G)$ is $\mathfrak{U}$-hypercentral in $G$, we can apply $[4, \mathrm{IV}, 6.10]$ to conclude that $G / \mathrm{C}_{G}\left(\mathrm{~F}^{*}(G)\right) \in \mathfrak{U}$. By $[9, \mathrm{X}, 13.12]$, $\mathrm{C}_{G}\left(\mathrm{~F}^{*}(G)\right) \leq \mathrm{F}(G)$ and so $G / \mathrm{F}(G) \in \mathfrak{U}$. Since every $G$-chief factor of $\mathrm{F}(G)$ is of order $p$, it follows that $G \in \mathfrak{U}_{p}$, contrary to supposition. Hence we have $G=\mathrm{F}^{*}(G)$.

Step 5. $G / \mathrm{Z}(G)$ is non-abelian simple and $G$ is perfect.

By Step $4 G=\mathrm{F}^{*}(G)=\mathrm{F}(G) \mathrm{E}(G)$, where $\mathrm{E}(G)$ is the layer of $G$, that is, the product of all components of $G$ (cf. [9, X, 13.18]). Since $G$ is not nilpotent, we have that $\mathrm{E}(G)$ is not contained in $\mathrm{F}(G)$. Let $H$ be a component of $G$. Then $H$ is normal in $G$ and $H / \mathrm{Z}(H)$ is non-abelian simple. Moreover, by Step 2, $p$ divides the order of $H$. In particular, $H$ is not $p$-supersoluble. By Step $1, H=G$.

Step 6. The conclusion of the proof.

Assume that $M$ is a maximal subgroup of $G$ not containing $\mathrm{Z}(G)$. Then $M Z(G)=G$ and so $M$ is a normal subgroup of $G$ because $M$ is normalised by $M$ and centralised by $\mathrm{Z}(G)$. But then $G / M$ is isomorphic to the abelian group $\mathrm{Z}(G) /(M \cap \mathrm{Z}(G))$, which contradicts that $G$ is perfect. Consequently $\mathrm{Z}(G)$ is contained in the Frattini subgroup of $G$. Let $A$ be a normal subgroup of $G$ such that $G / A$ is a chief factor of $G$. Then $A Z(G) \neq G$. Therefore $A$ contains $\mathrm{Z}(G)$ and so $A=\mathrm{Z}(G)$. Hence $\mathrm{Z}(G)$ belongs to all the chief series of $G$. Let $x$ be an element of $G$ of order $p$ or 4 . We know $\langle x\rangle$ is a partial CAP-subgroup of $G$. Hence $\langle x\rangle$ covers or avoids the $G$-chief factor $G / \mathrm{Z}(G)$. This implies that $x \in \mathrm{Z}(G)$. Applying [8, IV, 5.5], $G$ is $p$-nilpotent. This final contradiction completes the proof. 
Proof of Theorem 1.3. Clearly we may assume that $H \neq 1$. Let $p$ be a prime dividing the order of $\mathrm{F}^{*}(H)$. By hypothesis, every cyclic subgroup of $\mathrm{F}^{*}(H)$ of prime order or order 4 is a partial CAP-subgroup of $G$. By Lemma $2.1(1)$, every cyclic subgroup of $\mathrm{F}^{*}(H)$ of prime order or order 4 is a partial CAP-subgroup of $\mathrm{F}^{*}(H)$. By Theorem 1.4, we know that $\mathrm{F}^{*}(H)$ is $p$-supersoluble for all primes $p$. Therefore $\mathrm{F}^{*}(H)$ is supersoluble and so $\mathrm{F}^{*}(H)=\mathrm{F}(H)$. Moreover, applying Theorem 2.3, every Sylow subgroup of $\mathrm{F}(H)$ is contained in the supersoluble hypercentre of $G$. Hence $\mathrm{F}(H) \leq$ $\mathrm{Z}_{\mathfrak{U}}(G)$. By virtue of $[4, \mathrm{IV}, 6.10], G / \mathrm{C}_{G}(\mathrm{~F}(H)) \in \mathfrak{U}$. Since $G / H$ belongs to $\mathfrak{F}$ and $\mathfrak{F}$ contains $\mathfrak{U}$, it follows that $G / \mathrm{C}_{H}(\mathrm{~F}(H)) \in \mathfrak{F}$. This implies that $G / \mathrm{F}(H) \in \mathfrak{F}$ because $\mathrm{C}_{H}\left(\mathrm{~F}^{*}(H)\right) \leq \mathrm{F}(H)$ (cf. [9, X, 13.12]). Since every chief factor of $G$ below $\mathrm{F}(H)$ is of prime order and $\mathfrak{F}$ contains $\mathfrak{U}$, we obtain that $G$ acts $\mathfrak{F}$-hypercentrally on $\mathrm{F}(H)$. It follows that $G \in \mathfrak{F}$.

Proof of Corollary 1.5. We argue by induction on the order of $G$. Applying Lemma 2.1 (1), every cyclic subgroup of $\mathrm{F}_{p}^{*}(N)$ of order $p$ or 4 is a partial CAP-subgroup of $\mathrm{F}_{p}^{*}(N)$. Applying Theorem $1.4, \mathrm{~F}_{p}^{*}(N)$ is $p$-supersoluble. If $\mathrm{F}_{p}^{*}(N)$ is a $p^{\prime}$-group, then $\mathrm{F}_{p}^{*}(N)=\mathrm{O}_{p^{\prime}}(N)$ and so $N$ is a $p^{\prime}$-group. In this case $G \in \mathfrak{E}_{p^{\prime}} \mathfrak{F}=\mathfrak{F}$. Therefore, we may assume that $p$ divides the order of $\mathrm{F}_{p}^{*}(N)$. Since every abelian chief factor in a given chief series of $\mathrm{F}_{p}^{*}(N)$ with order divisible by $p$ is central in $\mathrm{F}_{p}^{*}(N)$, we conclude that $\mathrm{F}_{p}^{*}(N)$ is $p$-nilpotent, that is, $\mathrm{F}_{p}^{*}(N)=\mathrm{O}_{p^{\prime}, p}(N)$. Set $\bar{G}=G / \mathrm{O}_{p^{\prime}}(G)$ and $\bar{N}=N \mathrm{O}_{p^{\prime}}(G) / \mathrm{O}_{p^{\prime}}(G)$. Clearly $\mathrm{F}_{p}^{*}(\bar{N})=\mathrm{F}_{p}^{*}(N) \mathrm{O}_{p^{\prime}}(G) / \mathrm{O}_{p^{\prime}}(G)$. If $\bar{L}$ is a cyclic subgroup of $\mathrm{F}_{p}^{*}(\bar{N})$ of order $p$ or 4 , we can write $\bar{L}=L \mathrm{O}_{p^{\prime}}(G) / \mathrm{O}_{p^{\prime}}(G)$, where $L$ is a cyclic subgroup of $\mathrm{F}_{p}^{*}(N)$ of order $p$ or 4 . By hypothesis, $L$ is a partial CAP-subgroup of $G$.

Hence $\bar{L}$ is a partial CAP-subgroup of $\bar{G}$ by Lemma 2.1 (3). Thus $\bar{G}$ satisfies the hypothesis of the theorem. If $\mathrm{O}_{p^{\prime}}(G) \neq 1$, then $\bar{G} \in \mathfrak{F}$. Then $G \in \mathfrak{F}$, as $\mathfrak{E}_{p^{\prime}} \mathfrak{F}=\mathfrak{F}$. Hence we can assume that $\mathrm{O}_{p^{\prime}}(G)=1$. Therefore $\mathrm{F}^{*}(N)=$

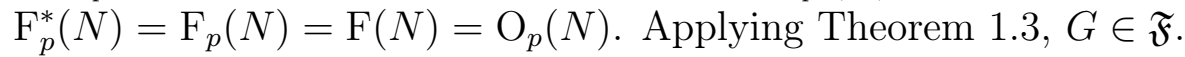

\section{Acknowledgements}

The first and the second authors have been supported by MEC, Spain, and FEDER, European Union (Grant No. MTM-2007-68010-C03-02) and MICINN, Spain (Grant No. MTM-2010-19938-C03-01). The third author has been supported in part by NSFC (Grant No. 11171353/A010201) and NSF of Guangdong (Grant No. S2011010004447). Part of this research was carried out during a visit of the third author to the Departament d'Àlgebra, Universitat de València, Burjassot, València, Spain, and the Institut Universitari de Matemàtica Pura i Aplicada, Universitat Politècnica de València, 
València, Spain, between September, 2009 and August, 2010. He is grateful to both institutions for their warm hospitality and, in particular, to the Universitat Politècnica de València for the financial support given via its Programme of Support to Research and Development 2010.

\section{References}

[1] A. Ballester-Bolinches and L. M. Ezquerro. Classes of Finite Groups, volume 584 of Mathematics and its Applications. Springer, New York, 2006 .

[2] A. Ballester-Bolinches, L. M. Ezquerro, and A. N. Skiba. Local embeddings of some families of subgroups of finite group. Acta Math. Sinica, English Series, 25:869-882, 2009.

[3] A. Ballester-Bolinches, L. M. Ezquerro, and A. N. Skiba. On second maximal subgroups of Sylow subgroups of finite groups. J. Pure Appl. Algebra, 215:705-714, 2011.

[4] K. Doerk and T. Hawkes. Finite Soluble Groups, volume 4 of De Gruyter Expositions in Mathematics. Walter de Gruyter, Berlin, New York, 1992.

[5] L. M. Ezquerro. A contribution to the theory of finite supersolvable groups. Rend. Sem. Mat. Univ. Padova, 89:161-170, 1993.

[6] Y. Fan, X. Guo, and K. P. Shum. Remarks on two generalizations of normality of subgroups. Chinese Ann. Math., 27A(2):169-176, 2006. Chinese.

[7] X. Guo and L. L. Wang. On finite groups with some semi cover-avoiding subgroups. Acta Math. Sin. (Engl. Ser.), 23(9):1689-1696, 2007.

[8] B. Huppert. Endliche Gruppen I, volume 134 of Grund. Math. Wiss. Springer Verlag, Berlin, Heidelberg, New York, 1967.

[9] B. Huppert and N. Blackburn. Finite groups III, volume 243 of Grund. Math. Wiss. Springer-Verlag, Berlin, 1982.

[10] Y. Li. On cover-avoiding subgroups of Sylow subgroups of finite groups. Rend. Sem. Mat. Univ. Padova, 123:249-258, 2010.

[11] Y. Li, L. Miao, and Y. Wang. On semi cover-avoiding maximal subgroups of Sylow subgroups of finite groups. Comm. Algebra, 37(4):11601169, 2009. 